\title{
The added value of three-dimensional ultrasonography in uterine pathology
}

\author{
MIHAELA GRIGORE ${ }^{1,2}$, RAZVAN POPOVICI $^{1,2}$, LOREDANA MARIA HIMINIUC $^{1,2}$, \\ IOANA SADIYE SCRIPCARIU ${ }^{1,2}$, BOGDAN FLORIN TOMA ${ }^{1,2}$, \\ ANA MARIA GRIGORE ${ }^{1}$, MIHAELA OANCEA ${ }^{3}$ and ROMEO MICU ${ }^{3}$ \\ ${ }^{1}$ Department of Obstetrics and Gynaecology, 'Grigore T. Popa' University of Medicine and Pharmacy, 700115 Iasi; \\ ${ }^{2}$ Clinical Hospital of Obstetrics and Gynaecology, 'Cuza Voda', 700038 Iasi; ${ }^{3}$ Department of Obstetrics and \\ Gynaecology, 'Iuliu Hatieganu’ University of Medicine and Pharmacy, 400012 Cluj-Napoca, Romania
}

Received May 31, 2021; Accepted June 30, 2021

DOI: $10.3892 /$ etm.2021.10696

\begin{abstract}
The rapid development achieved over the last decades in volume rendering of ultrasound data, known as three-dimensional (3D) ultrasound technique, leads to new opportunities for refining the diagnosis in many gynaecologic conditions. The aim of the present study was to evaluate the advantages of 3D ultrasound over two-dimensional (2D) ultrasound in uterine pathology and to establish the optimal time point during the menstrual cycle to perform 3D ultrasound examination in order to achieve the maximum of useful information. A cross-sectional study on 200 patients who underwent gynaecologic 2D and 3D ultrasound examinations was performed. The addition of $3 \mathrm{D}$ examination to $2 \mathrm{D}$ ultrasound in uterine pathology provided the most useful information concerning: Congenital uterine anomalies, intrauterine devices (IUDs), adenomyosis, and submucous myomas. The findings showed that the 3D ultrasound scan is a useful tool in gynaecology, especially in cases with congenital uterine anomalies, myoma, and IUD. Although initially it was used for research purposes only, recent findings suggest its usefulness in routine ultrasound scan and the possibility of witnessing its introduction as a recommended examination procedure in the foreseeable future. Further research should be conducted in order to establish the sensitivity of 3D ultrasound in the detection of minor endometrial conditions, by correlating the imaging findings with the hysteroscopic results.
\end{abstract}

\section{Introduction}

In the last few decades, significant progress has been made in the quality of imaging techniques in gynaecology that has

Correspondence to: Dr Razvan Popovici, Department of Obstetrics and Gynaecology, 'Grigore T. Popa' University of Medicine and Pharmacy, 16 Universitatii Street, 700115 Iasi, Romania E-mail: razpopovici@yahoo.com; razvan.popovici@umfiasi.ro

Key words: three-dimensional ultrasonography, congenital uterine anomalies, coronal plane, intrauterine device, myoma consistently contributed to the improvement and refining of diagnoses. Despite competition from magnetic resonance imaging (MRI) and computer tomography (CT), ultrasound remains the first-line modality for gynaecologic applications. The introduction of three-dimensional (3D) ultrasound in the early 1990s was an important step, and numerous studies have been conducted to determine the impact of 3D ultrasound (1). Currently, 3D is widely accepted in the diagnosis of foetal abnormalities and in the assessment of the main complications of pregnancy $(2,3)$. Few studies have been published in the field of gynaecologic applications of 3D ultrasound, rendering the present study a vital one.

The aim of the present study was to evaluate the advantages of 3D ultrasound over two-dimensional (2D) ultrasound in uterine pathology and to establish the optimal time point, with regards to the menstrual cycle, to perform 3D ultrasound examination in order to attain the maximum of useful information.

\section{Patients and methods}

Patient details. A cross-sectional study was performed in which 200 patients [age range, 19-76 years; mean, 40.38 years; inclusion and exclusion criteria provided in Table I] underwent gynaecologic and ultrasound examinations at Medis Medical Centre Iasi, Romania, between November 2019 and October 2020, comprising standard 2D followed by 3D ultrasound. For both examinations, a 3D endo-vaginal ultrasound probe [4-8 megahertz (MHz), Voluson E8; GE Healthcare] was used. The ultrasound examination began with a conventional 2D scan in order to establish the uterus position and its dimensions (longitudinal and antero-posterior diameters and the width of the corpus). Endometrial thickness was measured in the longitudinal plane, including the two layers at the level of its maximum thickness. Any pathological findings were recorded. The adnexal region was then scanned in order to identify the ovaries, the fallopian tube and a possible adnexal pathology. After the 2D ultrasound examination and Doppler assessment a diagnosis was established. The same sonographer, longitudinally along the length of the uterus, obtained the $3 \mathrm{D}$ volume. The coronal plane was obtained and then volume contrast imaging was gently applied (4). Both 2D images and 
$3 \mathrm{D}$ volumes were saved on the ultrasound machine's hard drive. The findings observed by 2D and 3D ultrasound were analysed by sonographers and compared. In cases with endometrial pathology, in order to confirm the diagnosis, a hysteroscopy (considered gold standard) was performed.

A comparison was made between the patients who benefitted from the reconstructed coronal view of the uterus vs. those who did not, based on the indication for scanning, and sonographic findings on the 2D ultrasonography.

Ethics. Each patient was properly informed regarding the conditions of the study and provided informed consent. Ethics approval was obtained from the Institutional Board of the 'Medis' Medical Centre (Iasi, Romania).

Statistical analysis. Data were analysed using the statistical package SPSS 26 (IBM Corp.) and JASP 0.13 (University of Amsterdam). The statistical tests used were: Chi Square, Shapiro-Wilk, Kruskal-Wallis, Levene and binomial test. The cut-off value of $\mathrm{P}<0.05$ was considered to indicate a statistically significant difference. The confidence interval was designated at $95 \%$.

\section{Results}

The age of the patients ranged between 19 and 76 years with a mean of 40.38 years (SD, 9.133). The indications for scanning for the 200 patients are presented in Table I.

In 71 cases (35.5\%) (CI 28.9-42.6\%) the ultrasound scan (both, 2D and 3D) revealed no uterine abnormalities. In 129 cases, different conditions were encountered. The results are presented in Table II.

The 3D ultrasound changed the diagnosis established by 2D ultrasound in 6 cases $(3.0 \%)$ (CI 1.1-6.7\%): Four cases with uterine congenital anomalies and two cases with IUD malposition in the uterine cavity.

In 53 cases $(26.5 \%)$ (CI 20.5-33.2\%), although the diagnosis was not changed it was completed by adding 3D and coronal plane examination. In cases with uterine myoma, the $3 \mathrm{D}$ ultrasound proved to be useful when myoma was situated intracavitary (total or partial) by showing the type of myoma (G0, 1 or 2 type), (Fig. 1A-C).

Uterine anomalies, classified as per the ESHRE/ESGE criteria (5) suspected by $2 \mathrm{D}$, but completely diagnosed by $3 \mathrm{D}$ ultrasound were as follows: Arcuate uterus, 3 cases; septate uterus, 4 cases (Fig. 2A-C); bicorn uterus, 1 case and unicorn uterus, 1 case.

In two cases with IUD, the diagnosis of misplaced or incongruence between IUD and uterine cavity was missed by 2D ultrasound (Fig. 3A-C).

High level of agreement was found between 2D and 3D in cases of uterine myoma and adenomyoss, a $\kappa$ value of 0.8 and 0.74 , respectively. A moderate level of agreement was observed in cases of polyps $(\kappa-0.67)$ and a low one in IUD $(\kappa-0.44)$.

In order to establish the optimal time point for performing a $3 \mathrm{D}$ scan with regards to menstrual cycle, results were compared in relation to the endometrial thickness. The quality of 3D images was favorable in 149 cases $(74.5 \%)(\mathrm{CI}, 67.9-80.4 \%)$ and inadequate in 51 cases $(25.5 \%)$ (CI: 19.6-32.1\%). The quality of the image was quantified by the possibility of obtaining a clear picture of the uterine cavity and endometro-myometrial junction (EMJ). The mean endometrial thickness was $7.86 \mathrm{~mm}$ (SD, 2.903) with values between 2 and $19 \mathrm{~mm}$. A significant statistical relationship existed between the quality of the image obtained and the thickness of the endometrium $(\mathrm{P}=0.622)$. A thin endometrium was associated with a poor 3D image. The present study showed a high probability to achieve a good image if the endometrium was $\geq 7.38 \mathrm{~mm}$ (value calculated using the Kruskal-Wallis non-parametric test).

\section{Discussion}

Ultrasonography is the most frequently used imaging technique in the assessment of the female genital tract. Usually the uterus and ovaries are evaluated using a 2D endovaginal ultrasonography. The main disadvantage of the $2 \mathrm{D}$ ultrasound scan consists in the difficulty of obtaining the coronal plane of the uterus. Adding a 3D ultrasound scan to the conventional examination may be beneficial as the coronal plane of the uterus can be obtained easily using the reconstruction $3 \mathrm{D}$ technique.

The present study revealed that from a total number of 200 examinations, 3D examination changed the diagnosis in 6 cases $(3.0 \%)$ (CI: $1.1-6.7 \%)$ and added useful information or reinforced the diagnosis in 53 cases (26.5\%) (CI: 20.5-33.2\%).

The most useful information was obtained in patients referred to for ultrasonic scan for the following indications: Infertility, uterine haemorrhage and IUD placement follow-up. Concerning the accuracy of the final diagnosis, the most useful information was obtained in patients with uterine pathology: Uterine congenital anomalies, IUD misplacement, adenomyosis, and submucous myomas. Neither 3D nor volume contrast imaging (VCI) were useful in cases with normal uterus.

Although conventional 2D ultrasound has a good accuracy in diagnosing congenital uterine anomalies, it is highly dependent on the expertise of the examiner and is limited by the difficulty in obtaining the coronal plane of the uterus. Several studies have demonstrated the advantages of 3D ultrasound in diagnosing uterine anomalies (6-8). Comparing 3D ultrasound to laparoscopy and hysteroscopy, Mohamed et al recorded a sensitivity of $97 \%$, specificity of $96 \%$, positive predictive value of $92 \%$ and negative predictive value of $99 \%$ in the diagnosis of uterine anomalies (9). Ghi et al reported both a sensitivity and a specificity of $100 \%$ in the diagnosis of uterine malformations and $96 \%$ concordance between ultrasound and endoscopy with respect to the type of anomaly diagnosed (10). In this study, 3D was mandatory for the final diagnosis in all cases with uterine congenital anomalies. As the coronal plane enables the visualization of not only the endometrial cavity, but also of the uterine fundus, 3D scan was necessary for the differential diagnosis between bicornuate and septate uterus. Moreover, in these cases, 3D proved to be superior to hysteroscopy. Hysteroscopy is able to confirm an anomaly of the uterine cavity but is not able to provide any information regarding the external contour of the uterus. In addition, in the coronal plane of the uterus obtained with 3D ultrasound it is possible to measure the size of the septum and classify the anomaly according to the ESHRE/ESGE classification system of female genital anomalies (5). Although MRI is considered the gold standard for congenital uterine anomalies, 
Table I. Indications for the ultrasound scan $(\mathrm{N}=200)$.

Data

Indication

No. of patients

Percentage

On demand at a routine gynaecological consult

IUD control

Abdominal pain, dysmenorrhea

Menorrhagia

16.5

Infertility

Menstrual disorders

Myoma, cyst

8.0

Total

IUD- intrauterine device.

Table II. The results provided by the ultrasound scan $(\mathrm{N}=200)$.

\begin{tabular}{lrr}
\hline & & Data \\
\cline { 2 - 3 } Diagnosis & No. of patients & Percentage \\
\hline No uterine abnormalities & 71 & 35.5 \\
Uterine myoma/myomas & 52 & 26 \\
Uterine congenital anomalies & 10 & 5.0 \\
IUD displacement - Cooper IUD & 1 & 0.5 \\
IUD displacement - levonorgestrel IUD & 2 & 1 \\
Endometrial polyps & 17 & 8.5 \\
Isthmocele & 15 & 7.5 \\
Adenomyosiys & 22 & 11 \\
Endometrial hyperplasia & 10 & 5 \\
Total & 200 & 100 \\
\hline
\end{tabular}

IUD, intrauterine device.

3D can provide the same type of information (11). However, in doubtful or complex cases, MRI should be performed, particularly for the assessment of the cervix and vagina (12). Thus, 3D ultrasound may be associated with 2D ultrasound in the diagnosis of uterine malformations.

Analysing the IUD cases from the current study, 3D was useful for both types: Copper IUD and levonorgestrel (LNG IUD). VCI proved to be particularly useful especially in cases with LNG IUD. Observations of this study confirm those of several studies performed in order to assess the role of 3D ultrasound in patients with IUD (13-16). These studies agree that the coronal view shows the entire device and its position within the uterus, in this way helping to identify the cause of pelvic pain and (or) bleeding in patients with an embedded IUD (14). Hösli et al demonstrated that 3D ultrasound offers the advantage of a better visualisation of LNG IUD and at the same time the assessment of uterine anomalies (13). The coronal plane is useful not only to detect the exact position of the IUD, but also to measure the uterine cavity and thus to establish the proper size of the IUD to be inserted in the uterus (17).
One interesting issue in gynaecology is the assessment of submucous myomas. It is important when diagnosing a myoma to know its level of extension in the uterine cavity. Moreover, before planning a hysteroscopic resection of a submucous myoma, it is necessary to know the type of myoma ( $\mathrm{G} 0,1$ or 2 type). Salim et al demonstrated that three-dimensional sonohysterography (3D-SIS) had a similar accuracy as hysteroscopy in classifying submucous fibroids (18). Lee et al demonstrated that 3D-SIS is reproducible among different observers for quantification of the percentage of a submucous fibroid protruding into the uterine cavity (19). Mavrelos et al demonstrated that 3D-SIS may be useful in predicting complete hysteroscopic resection of submucous myomas (20). Although findings of the present study show a high level of agreement between $2 \mathrm{D}$ and $3 \mathrm{D}$ ultrasound in the diagnosis of myoma $(\kappa=0.8)$, reconstructed coronal plane added useful information for planning the surgical management of the case.

Regarding adenomyosis, 2D ultrasound was useful in establishing the diagnosis by revealing the classical ultrasound signs including: Anechoic foci, heterogeneous myometrium, 
A



B



C

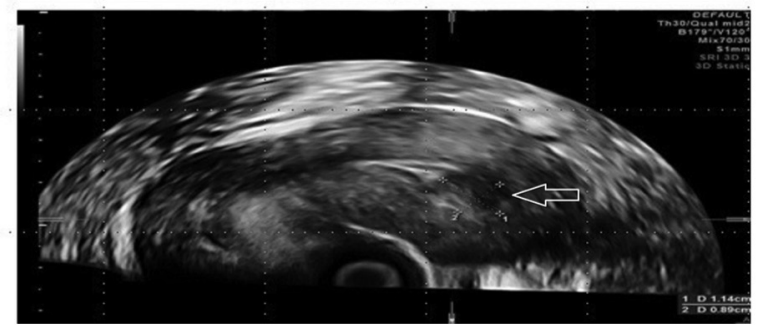

Figure 1. Submucous myoma (arrow). (A) 2D transversal plane of the uterus. (B) 2D ultrasound longitudinal plane of the uterus. (C) 3D ultrasound, coronal plane of the uterus. 2D, two dimensional; 3D, three dimensional.

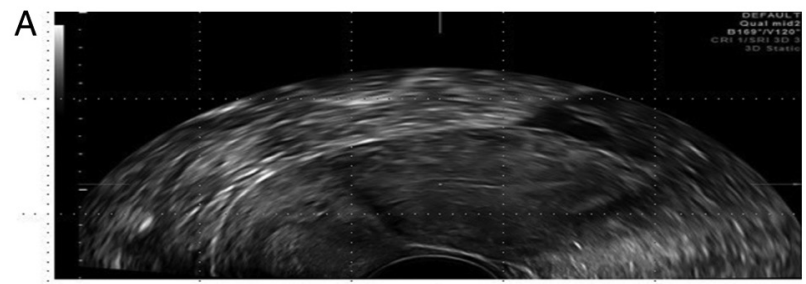

B

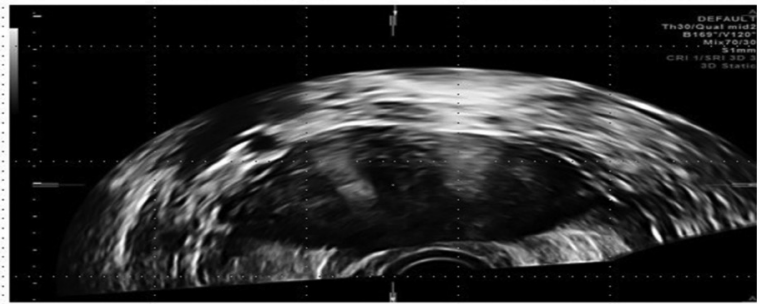

C

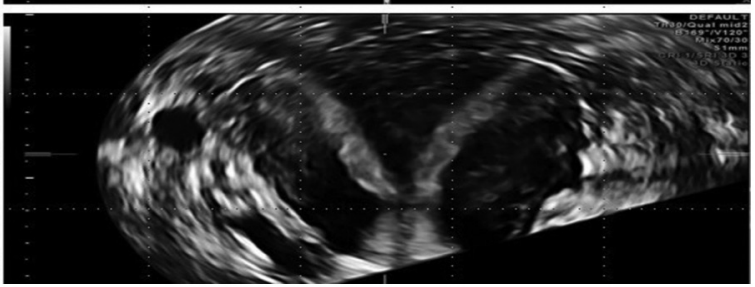

Figure 2. Septate uterus. (A) 2D ultrasound longitudinal plane of the uterus. (B) 2D transversal plane of the uterus. (C) 3D ultrasound, coronal plane of the uterus. 2D, two dimensional; 3D, three dimensional.

asymmetrical uterine wall, uterine enlargement and hyperechogenic striations (21). However, 2D ultrasound cannot provide information regarding the endometro-myometrial junction (EMJ). The changes in EMJ are considered a key element in diagnosing adenomyosis and traditionally the assessment of the EMJ has been part of the MRI evaluation
$A$



B

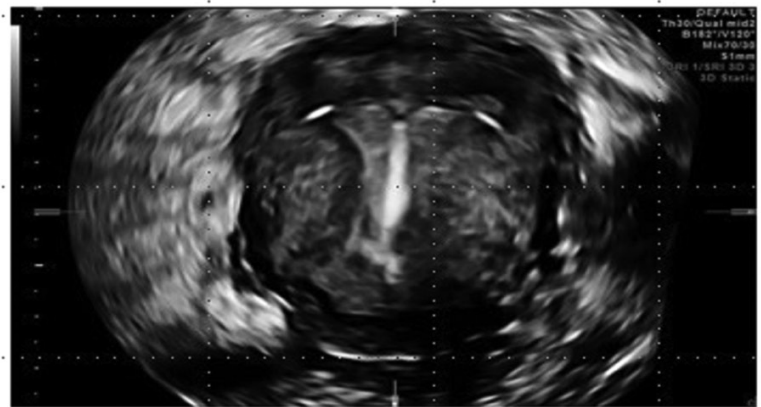

C.



Figure 3. Intracavitary IUD. (A and B) 2D ultrasound longitudinal plane and transversal plane showing a normal position of the IUD in the cavity. (C) 3D ultrasound with coronal plane showing the IUD embedded in the myometrium. 2D, two dimensional; 3D, three dimensional; IUD, intrauterine device.

of the uterus (21). 3D US enables visualisation of the coronal plane of the uterus and consequently provides a clear image of the EMJ. Due to the possibility of visualising the EMJ, $3 \mathrm{D}$ ultrasound opens up new horizons in the diagnosis of adenomyosis. Adding VCI to the coronal plane provides a clearer view of the EMJ. 3D ultrasound allows visualisation of the lateral and fundal EMJ, which is almost impossible to observe only with 2D imaging. Thanks to the clearer view provided by the VCI, this procedure is considered as the best in analysing and measuring the junctional zone (22). In our cases, 3D and VCI identified changes in EMJ as different size of the EMJ in different parts of the cavity and the penetration of the endometrium into the myometrium. There is some supposition that adenomyosis began with EMJ alteration and therefore 3D and VCI would be useful for an early diagnosis of the disease (23).

In the present study, the 2D ultrasound scan diagnosed endometrial polyps in 17 cases $(8.5 \%)$. In all cases the diagnosis was suspected by $2 \mathrm{D}$ ultrasound and confirmed by $3 \mathrm{D}$ and VCI. During the 2D ultrasound scan, a search for the pedicle artery sign was conducted in order to detect the 
position of the stalk of the polyp (24). 3D and VCI proved to be useful in 2 of 17 cases by providing a clearer image regarding the topography of the polyps. At the same time, the coronal plane was useful in the patient's understanding of the disease. Identical size of the polyps using 2D and 3D scans was obtained. Based on longitudinal and coronal plane and using Doppler mode we localised the base of the polyps and the place of its stalk. In all cases of polyps, hysteroscopy confirmed the diagnosis. 3D scan seems not to be superior to $2 \mathrm{D}$ in diagnosing endometrial polyps, but it helps in reinforcing the diagnosis and is very useful for the patient's understanding of the disease.

Another important issue concerns the question if there is a specific time frame regarding the endometrial cycle to perform a 3D ultrasound scan for obtaining the ideal image of the coronal plane. In relation to the size of the endometrium, the coronal plane was difficult to obtain if the size was $<7.38 \mathrm{~mm}$. Difficulties in obtaining a proper image of the uterine cavity have been noted in cases with endometrium thickness $<7.38 \mathrm{~mm}$ or in cases with multiple uterine myomas or adenomyosis. Benaceraff et al reported in a study on 66 patients that the coronal view is helpful in patients with an endometrium greater than or equal to $5 \mathrm{~mm}$ (14). Therefore, we recommend, in order to obtain a good 3D reconstructed image, that the sonography should be carried late in the follicular or in the secretory phase. In menopausal women, because the endometrial thickness is usually $<5 \mathrm{~mm}, 3 \mathrm{D}$ is not helpful.

Another advantage of 3D ultrasound that should be considered is the capability of storing volumes, ensuring a further process of the information. In this way, it is possible to follow-up the patients for a long period of time and if a uterine pathology will appear in the future, a reassessment of the previously rendered volumes would possibly bring useful information regarding the pathogenesis of the disease.

The main limitation of this study is represented by the fact that hysteroscopy was not performed in cases where 2D and 3D findings were normal; thus, it was possible to overlook some minor pathologic findings.

As a conclusion, we can state that the 3D ultrasound scan is an extremely useful tool in gynaecology, especially in cases with congenital uterine anomalies, submucous myomas and the assessment of IUD placement. Although initially it was used for research purposes only, currently it has been proven to be useful in the routine ultrasound scan and there is a possibility of witnessing its introduction as a mandatory examination procedure in the foreseeable future.

\section{Acknowledgements}

Not applicable.

\section{Funding}

No funding was received.

\section{Availability of data and materials}

All data generated or analysed during this study are included in this published article.

\section{Authors' contributions}

MG, RP, LMH and RM designed the study. MG, LMH, ISS and RP collected, analyzed and interpreted the patient data. MG, RM, MO, BFT, and AMG were responsible for discussion and interpretation of the data. BFT, AMG and ISS had major contributors in the writing of the manuscript. MG, RM, LMH and MO supervised and vizualised the final form. All authors have read and agreed to the published version of the manuscript. MG and RP confirms the authenticity of all raw data assesed in the manuscript.

\section{Ethics approval and consent to participate}

The present study was conducted in accordance with the World Medical Association Declaration of Helsinki and was approved by the Institutional Board of the 'Medis' Medical Centre Iasi, Romania (approval no. 12/18.09/2019). Informed consent was obtained from the patients.

\section{Patient consent for publication}

Not applicable.

\section{Competing interests}

The authors declare that there are no competing interests.

\section{References}

1. MerzE: Three-dimensional transvaginalultrasound in gynecological diagnosis. Ultrasound Obstet Gynecol 14: 81-86, 1999.

2. Turcan N, Bohiltea RE, Ionita-Radu F, Furtunescu F, Navolan D, Berceanu C, Nemescu D and Cirstoiu MM: Unfavorable influence of prematurity on the neonatal prognostic of small for gestational age fetuses. Exp Ther Med 20: 2415-2422, 2020.

3. Bohiltea R, Furtunescu F, Turcan N, Navolan D, Ducu I and Cirstoiu M: Prematurity and intrauterine growth restriction: Comparative analysis of incidence and short-term complication. In: Proceedings of SOGR 2018. The 17 National Congress of the Romanian Society of Obstetrics and Gynecology 2018: 708-712, 2019.

4. Abuhamad AZ, Singleton S, Zhao Y and Bocca S: The Z technique: An easy approach to the display of the mid-coronal plane of the uterus in volume sonography. J Ultrasound Med 25: 607-612, 2006.

5. Bermejo C, Martínez-Ten P, Ruíz-López L, Estévez M and Gil MM: Classification of uterine anomalies by 3-dimensional ultrasonography using ESHRE/ESGE criteria: Interobserver variability. Reprod Sci 25: 740-747, 2018.

6. Jurkovic D, Geipel A, Gruboeck K, Jauniaux E, Natucci M and Campbell S: Three-dimensional ultrasound for the assessment of uterine anatomy and detection of congenital anomalies: A comparison with hysterosalpingography and two-dimensional sonography. Ultrasound Obstet Gynecol 5: 233-237, 1995.

7. Salim R, Woelfer B, Backos M, Regan L and Jurkovic D: Reproducibility of three-dimensional ultrasound diagnosis of congenital uterine anomalies. Ultrasound Obstet Gynecol 21: 578-582, 2003.

8. Moini A, Mohammadi S, Hosseini R, Eslami B and Ahmadi F: Accuracy of 3-dimensional sonography for diagnosis and classification of congenital uterine anomalies. J Ultrasound Med 32: 923-927, 2013

9. Mohamed M, Momtaz MD, Alaa N, Ebrashy MD, Ayman A and Marzouk MD: Three-dimensional ultrasonography in the evaluation of the uterine cavity. MEFSJ 12: 41-46, 2007.

10. Ghi T, Casadio P, Kuleva M, Perrone AM, Savelli L, Giunchi S, Meriggiola MC, Gubbini G, Pilu G, Pelusi C and Pelusi G: Accuracy of three-dimensional ultrasound in diagnosis and classification of congenital uterine anomalies. Fertil Steril 92: 808-813, 2009 . 
11. Graupera B, Pascual MA, Hereter L, Browne JL, Úbeda B, Rodríguez I and Pedrero C: Accuracy of three-dimensional ultrasound compared with magnetic resonance imaging in diagnosis of Müllerian duct anomalies using ESHRE-ESGE consensus on the classification of congenital anomalies of the female genital tract. Ultrasound Obstet Gynecol 46: 616-622, 2015.

12. Bermejo C, Martinez Ten P, Cantarero R, Diaz D, Perez Pedregosa JP, Barrón E, Labrador E and López LR: Three-dimensional ultrasound in the diagnosis of Müllerian duct anomalies and concordance with magnetic resonance imaging. Ultrasound Obstet Gynecol 35: 593-601, 2010.

13. Hösli I, Holzgreve W and Tercanli S: Use of 3-dimensional ultrasound for assessment of intrauterine device position. Ultraschall Med 22: 75-80, 2001 (In German).

14. Benacerraf BR, Shipp TD and Bromley B: Three-dimensional ultrasound detection of abnormally located intrauterine contraceptive devices which are a source of pelvic pain and abnormal bleeding. Ultrasound Obstet Gynecol 34: 110-115, 2009.

15. Kalmantis K, Daskalakis G, Lymberopoulos E, Stefanidis K Papantoniou $\mathrm{N}$ and Antsaklis A: The role of three-dimensional imaging in the investigation of IUD malposition. Bratisl Lek Listy 110: 174-177, 2009.

16. Moschos E and Twickler DM: Does the type of intrauterine device affect conspicuity on 2D and 3D ultrasound? AJR Am J Roentgenol 196: 1439-1443, 2011.

17. Shipp TD, Bromley B and Benacerraf BR: The width of the uterine cavity is narrower in patients with an embedded intrauterine device (IUD) compared to a normally positioned IUD. J Ultrasound Med 29: 1453-1456, 2010.
18. Salim R, Lee C, Davies A, Jolaoso B, Ofuasia E and Jurkovic D: A comparative study of three-dimensional saline infusion sonohysterography and diagnostic hysteroscopy for the classification of submucous fibroids. Hum Reprod 20: 253-257, 2005.

19. Lee C, Salim R, Ofili-Yebovi D, Yazbek J, Davies A and Jurkovic D: Reproducibility of the measurement of submucous fibroid protrusion into the uterine cavity using three-dimensional saline contrast sonohysterography. Ultrasound Obstet Gynecol 28: 837-841, 2006.

20. Mavrelos D, Naftalin J, Hoo W, Ben-Nagi J, Holland T and Jurkovic D: Preoperative assessment of submucous fibroids by three-dimensional saline contrast sonohysterography. Ultrasound Obstet Gynecol 38: 350-354, 2011.

21. Dueholm M, Lundorf E, Hansen ES, Sørensen JS, Ledertoug S and Olesen F: Magnetic resonance imaging and transvaginal ultrasonography for the diagnosis of adenomyosis. Fertility Steril 76: 588-594, 2001.

22. Exacoustos C, Brienza L, Di Giovanni A, Szaboles B, Romanini ME, Zupi E and Arduini D: Adenomyosis: Three-dimensional sonographic findings of the junctional zone and correlation with histology. Ultrasound Obstet Gynecol 37: 471-479, 2011.

23. Naftalin J and Jurkovic D: The endometrial-myometrial junction: A fresh look at a busy crossing. Ultrasound Obstet Gynecol 34: $1-11,2009$.

24. Timmerman D, Verguts J, Konstantinovic ML, Moerman P, van Schoubroeck D, Deprest J and van Huffel S: The pedicle artery sign based on sonography with color Doppler imaging can replace second-stage tests in women with abnormal vaginal bleeding. Ultrasound Obstet Gynecol 22: 166-171, 2003. 